\title{
ON SIERPINSKI SETS
}

\author{
TOMEK BARTOSZYNSKI AND HAIM JUDAH
}

(Communicated by Andreas R. Blass)

\begin{abstract}
We prove that it is consistent with ZFC that every Sierpinski set is strongly meager. It is also proved that under $\mathrm{CH}$ every Sierpinski set is a union of two strongly meager sets.
\end{abstract}

\section{INTRODUCTION}

The purpose of this paper is to prove two theorems concerning the relationship between Sierpinski sets and strongly meager sets.

Definition. A set $S \subseteq \mathbf{R}$ is called a Sierpinski set if $S$ is uncountable and $S \cap H$ is countable for every measure zero set $H \subseteq \mathbf{R}$.

A set $X \subseteq \mathbf{R}$ is called strongly meager if for every null set $H \subseteq \mathbf{R}$ there exists $x \in \mathbf{R}$ such that $(X+x) \cap H=\varnothing$.

The main problem of this paper was asked by F. Galvin:

Is every Sierpinski set strongly meager?

It should be noted that the "dual" question obtained from the above by replacing the word "meager" by "null" and vice versa has a positive answer. In other words every Lusin set has strong measure zero (see [M] for this and many related results).

We will show that the answer to Galvin's question is consistently positivethere exists a model of ZFC where every Sierpinski set is strongly meager.

Another very important question about these sets is the following:

Do strongly meager sets form an ideal ( $\sigma$-ideal)?

We will show that these two questions are closely related by showing that:

Theorem. Assume CH. Then every Sierpinski set is a union of two strongly meager sets.

Finally we show that the assumption of $\mathrm{CH}$ is not very restrictive-a positive answer to both questions under $\mathrm{CH}$ yields a positive answer in ZFC.

Received by the editors March 7, 1989 and, in revised form April 17, 1989.

1980 Mathematics Subject Classification (1985 Revision). Primary 04A20. 
1. Let us start with the following simple observation: If both questions can be settled under $\mathrm{CH}$ then they can be settled in ZFC alone. In other words we have the following:

Theorem 1. (a) Suppose that $Z F C+C H \vdash$ every Sierpinski set is strongly meager. Then every Sierpinski set is strongly meager.

(b) Suppose that $\mathrm{ZFC}+\mathrm{CH} \vdash$ strongly meager sets form an ideal. Then strongly meager sets form an ideal.

Proof. We prove only part (a)-the proof of (b) is exactly the same.

Suppose that (a) is not true. Let $M$ be a model of ZFC such that $M \vDash$ there exists a Sierpinski set which is not strongly meager. Let $\mathrm{P}$ be the $\sigma$-closed notion of forcing which collapses $2^{\omega}$ onto $\omega_{1}$. Let $G \subseteq \mathrm{P}$ be an $M$-generic filter. Then since $\mathrm{P}$ does not add new reals $M[G] \vDash \mathrm{ZFC}+\mathrm{CH}+$ there exists a Sierpinski set which is not strongly meager. Contradiction.

Now let us show that there exists a model where all Sierpinski sets are strongly meager. Of course one can take a model where there are no Sierpinski sets but this is not exactly what we want.

Theorem 2. It is consistent with ZFC that there are Sierpinski sets and all of them are strongly meager.

Proof. Let $M$ be any model of ZFC in which $2^{\omega}>\omega_{1}$ and Lebesgue measure is $2^{\omega}$-additive. For example $M$ can be a model for $\mathrm{MA}+\neg \mathrm{CH}$. Let $\mathrm{B}_{\omega_{1}}$ be the algebra adding simultaneously $\omega_{1}$ random reals. For $\alpha<\omega_{1}$ let $\mathrm{B}_{\alpha}$ be the subalgebra of $\mathrm{B}_{\omega_{1}}$ which adds $\alpha$ many random reals. Also denote by $\mathrm{B}$ the standard measure algebra. Let $G \subseteq \mathrm{B}_{\omega_{1}}$ be a generic filter over $M$. We will show that the model $N=M[G]$ has the required properties.

The following facts are well known (see $[\mathrm{J}]$ ):

Lemma 1. (a) For every $\alpha<\omega_{1}, \mathrm{~B}_{\alpha} \simeq \mathrm{B}$.

(b) For every $\alpha<\omega_{1}, M\left[G \cap \mathrm{B}_{\alpha}\right] \vDash M \cap \mathbf{R}$ does not have measure zero.

Lemma 2. $N \vDash$ there are no Sierpinski sets of size $>\omega_{1}$.

Proof. Suppose not and let $S$ be a Sierpinski set of size $>\omega_{1}$ in $N$. Since every element of $S$ has a $\mathrm{B}_{\omega_{1}}$-name which depends on countable many "coordinates" there is an uncountable set $S^{\prime} \subseteq S$ and $\alpha<\omega_{1}$ such that $S^{\prime} \in$ $M\left[G \cap \mathrm{B}_{\alpha}\right]$. Clearly $S^{\prime}$ is a Sierpinski set in $M\left[G \cap \mathrm{B}_{c}\right]$.

To get a contradiction it is enough to see that:

Lemma 3. For every $\alpha<\omega_{1}$

$$
N_{\alpha}=M\left[G \cap \mathrm{B}_{\alpha}\right] \vDash \text { Lebesgue measure is } 2^{\omega} \text {-additive. }
$$

Proof. Fix $\alpha<\omega_{1}$. By Lemma $1 N_{\alpha}=M\left[G \cap \mathrm{B}_{\alpha}\right]=M[r]$ where $r$ is a random real over $M$. Suppose that $\left\{H^{\beta}: \beta<\lambda<2^{\omega}\right\}$ is a family of Borel, null subsets of $2^{\omega}$ in $N_{\alpha}$. It is well known that for every Borel, null set $H$ in $M[r]$ there exists a Borel, null set $\hat{H} \subseteq 2^{\omega} \times 2^{\omega}$ such that

$$
H=(\hat{H})_{r}=\text { vertical section of } \hat{H} \text { on } r \text {. }
$$


Therefore we have a family of Borel, null subsets of $2^{\omega} \times 2^{\omega}\left\{\hat{H}^{\beta}: \beta<\lambda\right\} \subseteq M$. By the assumption that the Lebesgue measure is $2^{\omega}$-additive in $M$ there exists a Borel, null set $A \subseteq 2^{\omega} \times 2^{\omega}$ such that $\bigcup_{\beta<\lambda} \hat{H}^{\beta} \subseteq A$. In particular the section $(A)_{r}$ is a Borel, null set in $M[r]$ which covers $\bigcup_{\beta<\lambda}\left(\hat{H}^{\beta}\right)_{r}=\bigcup_{\beta<\lambda} H^{\beta}$.

Since the choice of a family of null sets was arbitrary it finishes the proof.

Lemma 3 clearly implies that there are no Sierpinski sets in $N_{\alpha}$, which finishes the proof of Lemma 2.

It should be noted here that there are Sierpinski sets in $N$. In particular the set of $\omega_{1}$ random reals added by the filter $G$ is the simplest example.

Now we can finish the proof of the theorem.

Lemma 4. $N \vDash$ every Sierpinski set is strongly meager.

Proof. Let $S \in N$ be a Sierpinski set and let $H$ be a Borel, null set. Since $H$ is coded by a real number we can find $\alpha<\omega_{1}$ such that $H \in N_{\alpha}$.

Let $S^{\prime}=\left\{x \in S: x\right.$ is not random over $\left.N_{\alpha}\right\}$. Using Lemma 3 we easily see that $S^{\prime}$ is a countable set.

Lemma 5. There exists $z \in M \cap \mathbf{R}$ such that $\left(z+S^{\prime}\right) \cap H=\varnothing$.

Proof. By Lemma 1 (b) we know that $M \cap \mathbf{R}$ is not of measure zero in $N_{\alpha}$ (and in $N$ ). On the other hand the set $S^{\prime}+H$ is of measure zero. Therefore there exists $z \in M \cap \mathbf{R}$ such that $z \notin S^{\prime}+H$. It is clear that this is an element we are looking for.

This finishes the proof of the theorem: let $z$ be the element from Lemma 5 . For $x \in S^{\prime}, z+x \notin H$ by Lemma 5 . On the other hand if $x \in S-S^{\prime}, x$ is a random real over $N_{\alpha}$. Since $z \in M \subseteq N_{\alpha}, z+x$ is random over $N_{\alpha}$ as well. Therefore $z+x \notin H$ for every $x \in S$.

Now we prove:

Theorem 2. Assume CH. Then every Sierpinski set is a union of at most two strongly meager sets.

Proof. Let $S \subseteq \mathbf{R}$ be a Sierpinski set. We want to find two strongly meager sets $S^{0}$ and $S^{1}$ such that $S=S^{0} \cup S^{1}$. Let $\left\{H_{\alpha}: \alpha<\omega_{1}\right\}$ be an enumeration of Borel, null sets such that $H_{\alpha} \subseteq H_{\beta}$ for $\alpha \leq \beta<\omega_{1}$, and for every null set $H \subseteq \mathbf{R}$ there is $\alpha<\omega_{1}$ such that $H \subseteq H_{\alpha}$.

We build a sequence $\left\{S_{\alpha}^{1}, S_{\alpha}^{2}: \alpha<\omega_{1}\right\}$ of countable subsets of $S$ having the following two properties:

$$
S=\bigcup_{\alpha<\omega_{1}} S_{\alpha}^{1} \cup S_{\alpha}^{2}
$$

and

${ }^{(* *)} \quad$ for every $\alpha<\omega_{1}$ there are $z_{\alpha}^{1}, z_{\alpha}^{2} \in \mathbf{R}$ such that $S_{\beta}^{1} \cap\left(H_{\alpha}+\right.$ $\left.z_{\alpha}^{1}\right)=\varnothing$ and $S_{\beta}^{2} \cap\left(H_{\alpha}+z_{\alpha}^{2}\right)=\varnothing$ for all $\beta<\omega_{1}$. 
This would clearly finish the proof: let $S^{1}=\bigcup_{\alpha<\omega_{1}} S_{\alpha}^{1}$ and $S^{2}=\bigcup_{\alpha<\omega_{1}} S_{\alpha}^{2}$. By (*) $S=S^{1} \cup S^{2}$. Let $H \subseteq \mathbf{R}$ be a null set. Find $\alpha<\omega_{1}$ such that $H \subseteq H_{\alpha}$. By $\left(^{* *}\right), S^{1} \cap\left(H_{\alpha}+z_{\alpha}^{1}\right)=\varnothing$, hence $S_{1} \cap\left(H+z_{\alpha}^{1}\right)=\varnothing$. The same holds for the set $S^{2}$.

Therefore the problem is to define the sequence $\left\{S_{\alpha}^{1}, S_{\alpha}^{2}: \alpha<\omega_{1}\right\}$ having the properties above. The idea of the construction is the following:

Condition $\left(^{*}\right)$ will be taken care of using some kind of back and forth argument. For condition $\left.{ }^{* *}\right)$ we will use the following trick: to make sure that $S_{\beta}^{1} \cap\left(H_{\alpha}+z_{\alpha}^{1}\right)=\varnothing$ for $\beta \leq \alpha$ we will use the fact that $\bigcup_{\beta \leq \alpha} S_{\beta}^{1}$ is a countable set and we pick suitable real $z_{\alpha}^{1}$. In order to ensure that $S_{\beta}^{1} \cap\left(H_{\alpha}+z_{\alpha}^{1}\right)=\varnothing$ for $\beta>\alpha$ we will construct those sets in such a way that all elements of $S_{\beta}^{1}$ for $\beta>\alpha$ are random over some model containing $H_{\alpha}$ and $z_{\alpha}^{1}$.

The details of the construction are the following: We construct a sequence $\left\{S_{\alpha}^{1}, S_{\alpha}^{2}: \alpha<\omega_{1}\right\}$ together with an increasing sequence of countable, elementary substructures $\left\{M_{\alpha}, N_{\alpha}: \alpha<\omega_{1}\right\}$ of a suitably big part of the universe, say $H(\kappa, \in)$ for $\kappa>\left(2^{\omega}\right)^{+}$. From now on by model we will mean a structure as above.

For a structure $M$ containing $S$ let $S(M)=\{x \in S: x$ is not random over $M\}$. It is clear that the set $S(M)$ is countable if $M$ is countable. This is because in that case the set of reals which are not random over $M$ is null and $S$ is a Sierpinski set.

Before the general definition we will describe a few steps of the construction. Let $M_{0}$ be a countable model containing $H_{0}$ and $S$. Define

$$
S_{0}^{1}=S\left(M_{0}\right) \cap H_{0} \quad \text { and } \quad S_{0}^{2}=S\left(M_{0}\right)-S_{0}^{1} .
$$

We have to find reals $z_{\alpha}^{1}, z_{\alpha}^{2} \in \mathbf{R}$ satisfying condition (**). Notice that $S_{0}^{1}=$ $S \cap H_{0}$ is a countable set in $M_{0}$ and therefore we can pick $z_{0}^{1}$ to be any element of $M_{0}$ which does not belong to $S_{0}^{1}+H_{0}$. In order to find $z_{0}^{2}$ we have to work outside $M_{0}$. Let $N_{0}$ be a countable model containing $M_{0}$ such that $N_{0} \vDash " S\left(M_{0}\right)$ is a countable set in $N_{0}$ ". Again we easily find a real $z_{0}^{2}$ in $N_{0}$ satisfying $\left({ }^{* *}\right)$. Notice that at that point we partitioned the set $S\left(M_{0}\right)$ into two pieces. Every other element of $S$ is random over $M_{0}$ so $\left({ }^{* *}\right)$ is satisfied for the set $H_{0}$ and set $S_{\alpha}^{1}$ no matter how they are defined. This is due to the fact that if $x$ is a random real over a model $M$ and $z \in M$ is a real then $x+z$ is a random real over $M$, so in particular is not in $H_{0}$. Now in $N_{0}$ there are probably some new elements of $S$. To take care of them, let $M_{1}$ be a countable model containing $N_{0}$ and $H_{1}$ such that $M_{1} \vDash " S\left(N_{0}\right)$ is a countable set in $M_{1}$ ".

Let $S_{1}^{1}=\left(S\left(M_{1}\right) \cap H_{1}\right) \cup\left(S\left(N_{0}\right)-S\left(M_{0}\right)\right)$ and $S_{1}^{2}=\left(S\left(M_{1}\right)-S_{1}^{1}\right)-S\left(M_{0}\right)$. By the same argument as above (applied to $S_{0}^{1} \cup S_{1}^{1}$ ) we get a real $z_{1}^{1} \in M_{1}$ 
satisfying $\left({ }^{* *}\right)$. To get $z_{1}^{2}$ we construct a model $N_{1}$ containing $M_{1}$ such that $N_{1}$ ₹ " $S\left(M_{1}\right)$ is a countable set in $N_{1}$ ", and so on.

In general suppose that the sets $S_{\beta}^{1}, S_{\beta}^{2}$, reals $z_{\beta}^{1}, z_{\beta}^{2}$ and models $M_{\beta}$, $N_{\beta}$ have already been constructed for $\beta<\alpha<\omega_{1}$. In addition assume that $\bigcup_{\xi<\gamma} S_{\xi}^{1} \cup S_{\xi}^{2}=S\left(M_{\gamma}\right)$ for $\gamma<\beta<\alpha$. We have to find $S_{\alpha}^{1}, S_{\alpha}^{2}, z_{\alpha}^{1}, z_{\alpha}^{2}$ and $M_{\alpha}, N_{\alpha}$. Let $M_{\alpha}$ be a countable model such that

1. $M_{\beta}, N_{\beta} \subseteq M_{\alpha}$ for $\beta<\alpha$,

2. $M_{\alpha} \vDash " \bigcup_{\beta<\alpha} S\left(N_{\beta}\right)$ is a countable set in $M_{\alpha}$ ",

3. $H_{\alpha} \in M_{\alpha}$.

Consider the set $T=S\left(M_{\alpha}\right)-\bigcup_{\beta<\alpha} S\left(M_{\beta}\right)$. Sets $S_{\alpha}^{1}$ and $S_{\alpha}^{2}$ will be obtained by partitioning $T$ into two pieces. We have two cases:

Case 1. $\alpha$ is a limit ordinal. In this case let $S_{\alpha}^{1}=T \cap H_{\alpha}$ and $S_{\alpha}^{2}=T-S_{\alpha}^{1}$. We easily see that $M_{\alpha} \vDash$ " $S_{\alpha}^{1}$ is countable" and therefore we can find $z_{\alpha}^{1} \in M_{\alpha} \cap \mathbf{R}$ such that

$$
\bigcup_{\beta<\alpha} S_{\beta}^{1} \cap\left(H_{\alpha}+z_{\alpha}^{1}\right)=\varnothing .
$$

Let $N_{\alpha}$ be a model containing $M_{\alpha}$ such that $N_{\alpha} \vDash$ " $T$ is a countable set in $N_{\alpha}$ ". The same argument as above gives us $z_{\alpha}^{2} \in N_{\alpha} \cap \mathbf{R}$ with the property that

$$
\bigcup_{\beta<\alpha} S_{\beta}^{2} \cap\left(H_{\alpha}+z_{\alpha}^{2}\right)=\varnothing .
$$

Case 2. $\alpha$ is a successor ordinal, say $\alpha=\beta+1$. Notice that in this case $T$ is equal to $S\left(M_{\alpha}\right)-S\left(M_{\beta}\right)=\left(S\left(M_{\alpha}\right)-S\left(N_{\beta}\right)\right) \cup\left(S\left(N_{\beta}\right)-S\left(M_{\beta}\right)\right)$. Define

$$
S_{\alpha}^{1}=\left(T \cap H_{\alpha}\right) \cup\left(S\left(N_{\beta}\right)-S\left(M_{\beta}\right)\right) \text { and } S_{\alpha}^{2}=T-S_{\alpha}^{1} .
$$

In exactly the same way as above we find $z_{\alpha}^{1}, N_{\alpha}$ and then $z_{\alpha}^{2}$.

We have to check that conditions $\left({ }^{*}\right)$ and $\left({ }^{* *}\right)$ are satisfied. Notice that $\bigcup_{\xi<\gamma} S_{\xi}^{1} \cup S_{\xi}^{2}=S\left(M_{\gamma}\right)$ for $\gamma<\omega_{1}$. Therefore

$$
\bigcup_{\alpha<\omega_{1}} S_{\alpha}^{1} \cup S_{\alpha}^{2}=S \quad \text { since } \bigcup_{\alpha<\omega_{1}} S\left(M_{\alpha}\right)=S \text {. }
$$

For $\left(^{* *}\right)$ observe that for $\beta \leq \alpha<\omega_{1}, S_{\beta}^{1} \cap\left(H_{\alpha}+z_{\alpha}^{1}\right)=\varnothing$ by the choice of $z_{\alpha}^{1}$. On the other hand if $\beta>\alpha$ then $S_{\beta}^{1} \cap S\left(M_{\alpha}\right)=\varnothing$, which means that elements of $S_{\beta}^{1}$ are random reals over $M_{\alpha}$. Hence $S_{\beta}^{1} \cap\left(H_{\alpha}+z_{\alpha}^{1}\right)=\varnothing$ because $z_{\alpha}^{1} \in M_{\alpha} \cap \mathbf{R}$. The case of the other sequence is very similar: $S_{\beta}^{2} \cap\left(H_{\alpha}+z_{\alpha}^{2}\right)=\varnothing$ for $\beta \leq \alpha<\omega_{1}$ by the choice of $z_{\alpha}^{2}$. For $\beta>\alpha$ we show that elements of $S_{\beta}^{1}$ are random over $N_{\alpha}$ and the above argument applies.

Remark. Notice that small modification of the above argument yields that if the ideal of null sets has a basis of size $\omega_{1}$ then every Sierpinski set can be decomposed into two strongly meager sets. 


\section{REFERENCES}

[J] T. Jech, Set theory, Academic Press, 1978.

[M] A. Miller, Special subsets of the real line, in Handbook of Set Theoretical Topology, NorthHolland, Amsterdam, 1984.

Department of Mathematics, University of California, Davis, California 95616

Department of Mathematics, University of California, Berkeley, California 94720 